\title{
Estudo epidemiológico de idosos acometidos por intoxicação medicamentosa no estado da Paraíba: o retrato de uma década
}

\author{
Epidemiological study of elderly people affected by drug intoxication in the state of Paraíba: a \\ portrait of a decade \\ Estudio epidemiológico de ancianos afectados por envenenamiento por drogas en el estado de \\ Paraíba: retrato de una década
}

Recebido: 22/07/2021 | Revisado: 30/07/2021 | Aceito: 03/08/2021 | Publicado: 08/08/2021

Wagner Bernardo da Silva ORCID: https://orcid.org/0000-0003-0544-2193 Universidade Federal de Campina Grande, Brasil E-mail: wagner.bernardo@estudante.ufcg.edu.br Beatriz Maria da Conceição Murilo ORCID: https://orcid.org/0000-0001-6630-4566 Universidade Federal de Campina Grande, Brasil E-mail: biarebelde2016@gmail.com

Pedro Ithalo Francisco da Silva ORCID: https://orcid.org/0000-0002-8976-2591 Universidade Federal de Campina Grande, Brasil

E-mail: pedro.ithalo@estudante.ufcg.edu.br Ival da Costa Filho ORCID: https://orcid.org/0000-0003-4070-5972 Universidade Federal de Campina Grande, Brasil E-mail: ivalfilho09@gmail.com

Francisco Patrício de Andrade Júnior ORCID: https://orcid.org/0000-0003-0681-8439 Universidade Federal da Paraíba, Brasil

E-mail: juniorfarmacia.ufcg@outlook.com

Cinthya Maria Pereira de Souza ORCID: https://orcid.org/0000-0002-0153-9097

Centro Universitário UNIFACISA, Brasil E-mail: cinthyampsouza@ hotmail.com

\begin{abstract}
Resumo
Este trabalho objetivou investigar o perfil epidemiológico de idosos acometidos por intoxicação medicamentosa na Paraíba, entre os anos de 2007 a 2017. Tratou-se de um estudo epidemiológico, observacional, retrospectivo, analítico e documental, do tipo quantitativo, em que os dados foram coletados a partir de consultas à base de dados do Sistema de Informações de Agravos de Notificação (SINAN), disponibilizado pelo Departamento de Informática do Sistema Único de Saúde (DATASUS). Foram analisadas as variáveis como ano, cidade de notificação, sexo, faixa etária, escolaridade, circunstâncias do tipo de intoxicação e evolução dos casos. Para a análise das associações entre as variáveis aplicou-se o teste Qui-Quadrado de Independência, em que $p<0,05$ foi considerado estatisticamente significativo para as hipóteses de nulidade. Detectou-se que foram notificados 219 casos de intoxicação medicamentosa, sendo o ano de 2014 o mais prevalente $(21,46 \%)$ e João Pessoa foi a cidade com mais casos. Ao associar sexo e as outras variáveis não se observou significância estatística. O perfil epidemiológico de acometidos foi majoritariamente de indivíduos do sexo feminino (63,0\%), com 60 a 69 anos de Idade (55,0\%), sendo que a maioria dos casos relatados foram curados sem sequela $(72,6 \%)$. Destaca-se que os dados desta pesquisa podem ser utilizados para a criação de políticas públicas ou indicadores com o intuito de atender a população mais acometida.
\end{abstract}

Palavras-chave: Eventos adversos dos Fármacos; Toxicidade de drogas; Senilidade.

\begin{abstract}
This study aimed to investigate the epidemiological profile of elderly people affected by drug intoxication in Paraíba, between the years 2007 to 2017. It was an epidemiological, observational, retrospective, analytical and documentary study, of the quantitative type, in which data were collected from queries to the database of the Information System for Notifiable Diseases (SINAN), made available by the Informatics Department of the Unified Health System (DATASUS). Variables such as year, city of notification, sex, age, education, circumstances of the type of poisoning and evolution of cases were analyzed. For the analysis of the associations between the variables, the Chi-Square Test of Independence was applied, in which $\mathrm{p}<0.05$ was considered statistically significant for the null hypothesis. It was
\end{abstract}


found that 219 cases of drug intoxication were reported, with 2014 being the most prevalent year (21.46\%) and João Pessoa was the city with the most cases. When associating sex and other variables, no statistical significance was observed. The epidemiological profile of those affected was mostly female (63.0\%), aged 60 to 69 years $(55.0 \%)$, and most reported cases were cured without sequelae (72.6\%). It is noteworthy that the data from this research can be used to create public policies or indicators in order to serve the most affected population.

Keywords: Adverse effects of drugs; Drug toxicity; Senility.

\section{Resumen}

Este estudio tuvo como objetivo investigar el perfil epidemiológico de los ancianos afectados por intoxicación por drogas en Paraíba, entre los años 2007 a 2017. Fue un estudio epidemiológico, observacional, retrospectivo, analítico y documental, de tipo cuantitativo, en el que se recolectaron datos de consultas a la base de datos del Sistema de Información de Enfermedades Notificables (SINAN), puesta a disposición por el Departamento de Informática del Sistema Único de Salud (DATASUS). Se analizaron variables como año, ciudad de notificación, sexo, edad, escolaridad, circunstancias del tipo de intoxicación y evolución de los casos. Para el análisis de las asociaciones entre las variables se aplicó el Test Chi-Cuadrado de Independencia, en el cual p <0.05 se consideró estadísticamente significativo para la hipótesis nula. Se encontró que se reportaron 219 casos de intoxicación por drogas, siendo 2014 el año de mayor prevalencia $(21,46 \%)$ y João Pessoa fue la ciudad con más casos. Al asociar sexo y otras variables, no se observó significación estadística. El perfil epidemiológico de los afectados fue mayoritariamente femenino $(63,0 \%)$, de 60 a 69 años $(55,0 \%)$, y la mayoría de los casos notificados se curaron sin secuelas $(72,6 \%)$. Es de destacar que los datos de esta investigación se pueden utilizar para crear políticas públicas o indicadores con el fin de atender a la población más afectada.

Palabras clave: Efectos adversos de las drogas; Toxicidad farmacológica; Senilidad.

\section{Introdução}

Segundo a Organização Mundial da Saúde, uma das maiores vitórias da humanidade é o envelhecimento da população, pois hoje em dia o ser humano pode esperar viver até 60 anos ou mais, entretanto é também um dos maiores desafios a ser enfrentado pela sociedade, por causa do alto risco com a saúde desses indivíduos (OMS, 2015). Devido a esse fato, na última década, as demandas sociais e econômicas do mundo aumentaram, contudo os idosos são ignorados e muitas vezes submetidos ao descaso (Miranda, Mendes \& Silva, 2019).

No Brasil, a população idosa está em constante crescimento. De acordo com os dados do Instituto Brasileiro de Geografia e Estatística (IBGE) de 2018, esse grupo etário representava 13,5\% da população com 28 milhões de indivíduos (Perissé \& Marli, 2019). Em projeções futuras, no ano de 2031 serão 43,2 milhões de idosos, superando o número de crianças e adolescentes pela primeira vez, e no ano de 2050 seremos o quinto país em relação a população, com um contingente de 254 milhões habitantes, perdendo apenas para países como Índia, China, Estados Unidos e Indonésia (Santos \& Santos, 2019; Mendes et al, 2018).

Portanto, devido ao aumento dessa população, será um grande desafio para a geriatria prevenir e tratar problemas de saúde que aparecem com a idade, como a imobilidade, instabilidade, incontinência urinária, insuficiência cerebral e devido ao uso irracional de medicamentos (Maia et al., 2011). Os idosos apresentam um organismo debilitado, por causa da idade avançada, o que acarreta na utilização de vários medicamentos de divergentes classes farmacêuticas para tratar as doenças, porém muitos utilizam irracionalmente sem a orientação de um profissional da saúde (Pereira et al., 2019).

O uso indiscriminado de várias classes de medicamentos pode causar intoxicações decorrentes de uma não indicação farmacológicas de doses elevadas, de medicamentos não prescritos ou de quando o indivíduo faz uso de medicamentos por período prolongado. As intoxicações são consideradas um problema de saúde pública contribuindo para 5\% das admissões hospitalares no país (Gotadelo, 2014).

Segundo Rangel e Francelino (2018) a maioria das intoxicações no Brasil no ano de 2010, foi por medicamentos, com 27,75\% dos casos, sendo a principal causa de óbitos no país (Rangel \& Francelino, 2018). Contudo, estudos epidemiológicos de intoxicação medicamentosa podem servir para uma reflexão nos padrões de consumo de medicamentos no país, fornecendo a criação e aprimoramento das políticas de vigilância sanitária (Mota, 2012). Devido a esse fato o objetivo deste trabalho foi de 
delinear perfil epidemiológico dos idosos acometidos por intoxicações medicamentosas no estado da Paraíba, entre os anos de 2007 a 2017.

\section{Metodologia}

Tratou-se de um estudo epidemiológico, observacional, retrospectivo, analítico e documental, do tipo quantitativo, uma vez que o objetivo do trabalho foi compreender os fenômenos através da coleta de dados numéricos, apontando preferências, comportamentos e outras ações dos indivíduos que pertencem a determinado grupo (Barros Lima et al., 2013).

Os dados foram coletados a partir de consultas à base de dados do Sistema de Informação de Agravos de Notificação (SINAN), disponibilizada pelo Departamento de Informática do Sistema Único de Saúde (DATASUS) nos casos de intoxicação exógena por medicamentos (Brasil, 2020). Foram analisadas as variáveis como ano, cidade de notificação, sexo, faixa etária, etnia, escolaridade, zona de residência, circunstâncias do tipo de intoxicação e evolução dos casos.

Optou-se por estudar o estado da Paraíba que está localizado na região nordeste do Brasil. Sua área territorial abrange $56.467,242 \mathrm{~km} 2$, com uma população estimada para o ano de 2019 de 4.018.127 de habitantes, com rendimento nominal mensal domiciliar per capita de R\$ 929. Em relação a saúde apresentou 2.622 estabelecimentos de saúde vinculados ao Sistema Único de Saúde (SUS) (Brasil, 2020).

Os dados foram compilados e analisados a partir do software Statistical Package for the Social Sciences, versão 13.0. Para a análise das associações entre as variáveis aplicou-se o teste Qui-Quadrado de Independência, em que p<0,05 foi considerado estatisticamente significativo para as hipóteses de nulidade.

O mapa da distribuição espacial foi elaborado com o software Qgis 3.10.14 utilizando a frequência das notificações nos municípios.

Contudo, como no estudo foram utilizados dados secundários públicos, disponíveis no SINAN e por não conter variáveis que possibilitem a identificação dos indivíduos estudados, o presente estudo dispensou a autorização do Comitê de Ética conforme estabelece a Resolução n 466, de 12 de dezembro de 2012, do Conselho Nacional de Saúde (CNS).

\section{Resultados e Discussão}

Durante os anos de 2007 a 2017 foram registrados 219 casos de intoxicação medicamentosa em idosos no estado da Paraíba. De acordo com a Figura 1, o ano mais prevalente foi o de 2014 com 21,5\% e sofreu uma queda com o passar dos anos, tendo em vista que o ano de 2017 teve 16,9\% dos casos. Os anos de 2007 e 2008 não apresentaram notificações, evidenciando a falta de melhorias no sistema de notificações. 
Figura 1. Casos de idosos acometidos por intoxicação medicamentosa na Paraíba, entre os anos de 2007 a 2017.

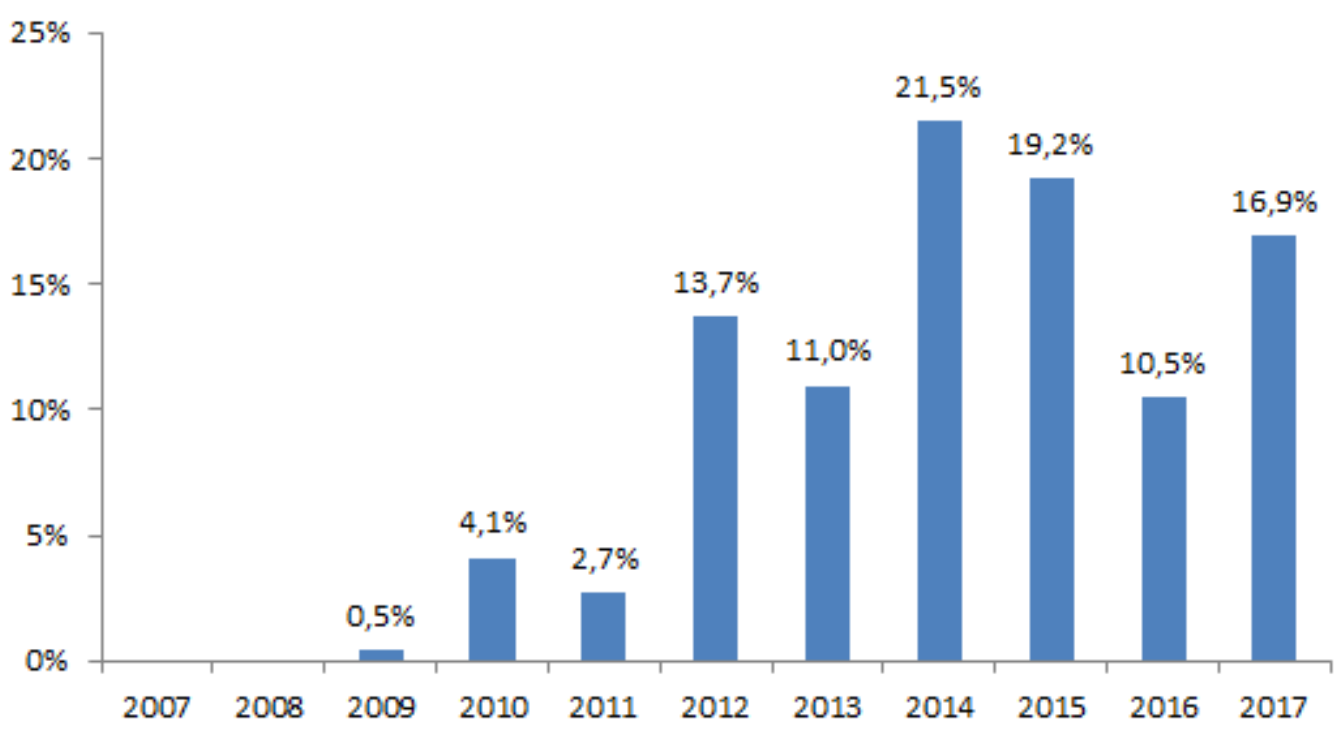

Fonte: Adaptado do SINAN (2021).

Na Paraíba, no ano de 2014, foram registradas 1.536 farmácias e drogarias, na rede privada e pública, e com passar dos anos esse número foi crescendo a ponto de em 2018 serem registradas 1.439 apenas no setor privado (IDME, 2014; CFF, 2019). Este fato pode contribuir para uma maior facilidade na compra de medicamentos pela população, o que dificulta cada vez mais a fiscalização dos órgãos competentes e consequentemente aumenta o número de casos de intoxicação (Silva et al., 2013).

Os casos subnotificados podem ser devido ao disfarce de dados, por causa de que muitas vezes o diagnóstico de intoxicação medicamentosa nos idosos é dificultado devido a saúde frágil dos mesmos (Rangel \& Francelino, 2018). A alta prevalência de medicamentos, a polifarmácia e o aumento progressivo de uso de medicamentos com a idade são fatores que predispõem esse grupo aumento do risco a agravos envolvendo medicamentos (Oliveira et al., 2017).

Os idosos compõem a faixa etária que mais utiliza medicamentos, devido a presença de várias doenças, somando esse fato com o quadro clínico cognitivo e as limitações físicas da idade, muitas das vezes a terapia medicamentosa não é eficaz e traz agravos à saúde (Medeiros, Soares \& Saatkamp, 2012). É visto que os medicamentos são utensílios mais importantes na saúde do idoso, porém além de tratar doenças crônicas também é um dos principais fatores de risco a essa população (Ramos et al.,2016).

No entanto, o uso irracional de medicamentos podem trazer consequências graves à saúde dos idosos, visto que é o grupo que tem mais tendência a apresentar problemas, pois estão sempre envolvidos a erros de dosagem, troca de medicamentos, presença de mais de um princípio ativo, interações, reações adversas e intoxicações, assim este grupo necessita de ajustes (Mastroianni et al., 2011).

Um fator que contribui para complicações referentes ao uso de medicamentos, é que com o envelhecimento as trocas fisiológicas, antes ocorridas de forma normal, agora podem alterar a distribuição, metabolismo e excreção de fármacos, pois modificam a ação dos mesmos e afetam a sua concentração no sítio receptor (Silva, Schmidt \& Silva, 2012). Os medicamentos de uso terapêutico estreito devem usados com cuidado, pois no idoso pode ter o risco de ocorrer sobredosagem devido às alterações na função renal, no fluxo sanguíneo e na distribuição hepática (Bitter, Schlender \& Woltersdorf, 2016).

Segunda Lima et al (2018) as classes de medicamentos mais utilizados por idosos são: anti-inflamatórios não esteroidais (AINEs), betabloqueadores, inibidores da enzima conversora de angiotensina (IECA), diuréticos, antidislipidémicos e depressores do SNC, ou seja, fármacos que são potencialmente interativos, além dos indutores (fenitoína, carbamazepina) e 
inhibidores enzimáticos como, por exemplo, cimetidina e omeprazol que, frequentemente, encontram-se envolvidos nas interações medicamentosas (IM) ameaçando a saúde do idoso (Lima et al., 2018).

Quanto ao percentual de notificações a nível municipal nos casos de intoxicação medicamentosa em idosos pode ser verificado na Figura 2 que a cidade de João Pessoa foi a que teve mais notificações de intoxicações, entre 19 à 127, seguido de Guarabira, Campina Grande e Cajazeiras, todas entre 8 a 19 casos. Este dado obtido é contrário ao de estudos como realizado no estado do Maranhão, em que a capital São Luís apresentou-se com minoria dos casos obtidos (Oliveira, Antunes \& Nicolina, 2018).

Figura 2. Distribuição Espacial dos casos de intoxicação medicamentosa nos municípios da Paraíba, entre os anos de 2007 a 2017.

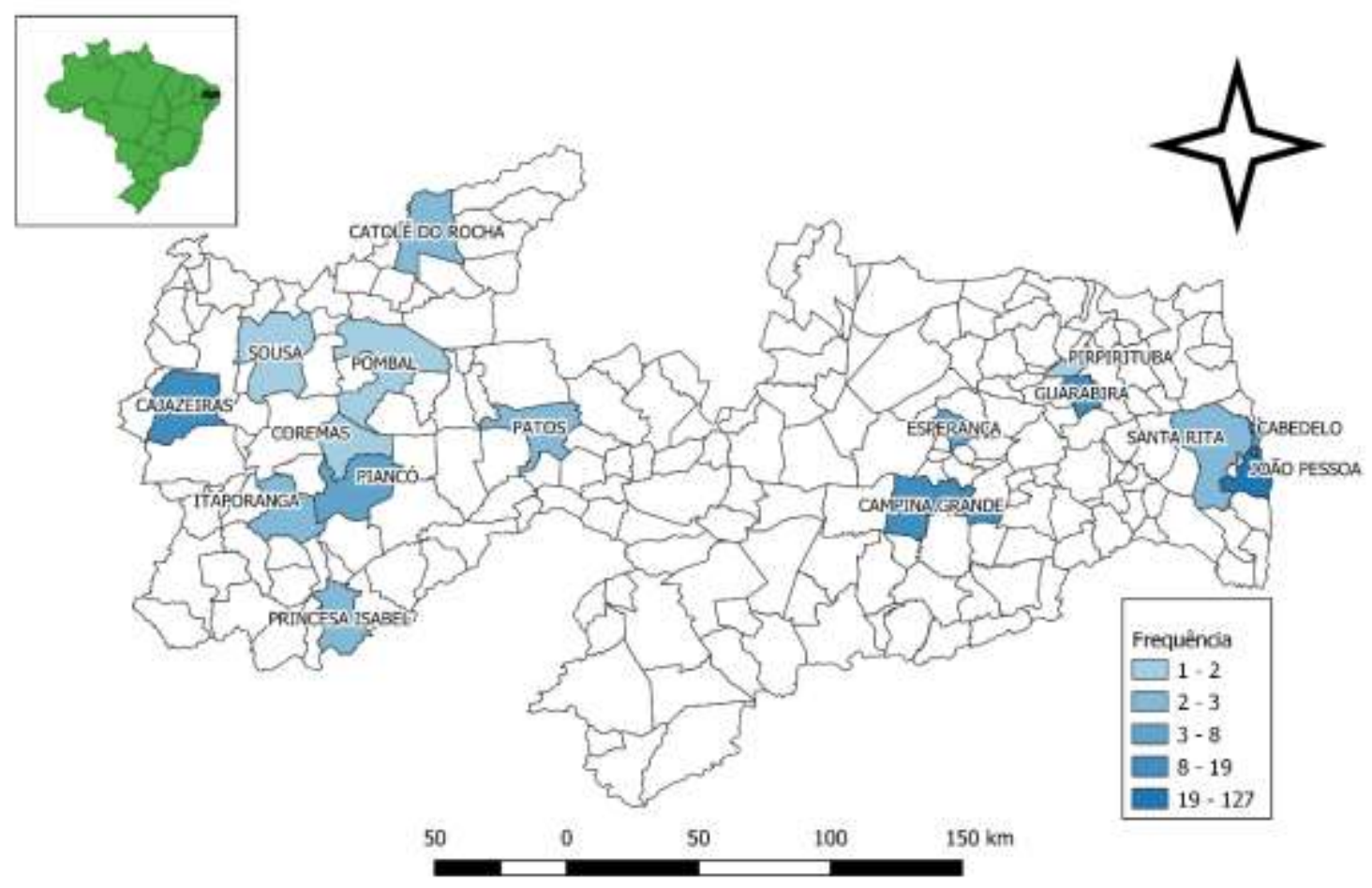

Fonte: Elaborado com Qgis (2021).

O fato de a capital João Pessoa ser majoritária, nos casos de intoxicação medicamentosa em idosos, se dá por ser a cidade com maior número de idosos em comparação com as outras cidades da Paraíba (Brasil, 2020).

Os dados referentes ao perfil dos acometidos de intoxicação medicamentosa podem ser observados na Tabela 1, como sexo, faixa etária, etnia, escolaridade e zona de residência, bem como suas associações. 
Tabela 1. Associação entre sexo e dados pessoais de idosos acometidos por Intoxicação medicamentosa na Paraíba, entre os anos de 2007 a 2017.

\begin{tabular}{|c|c|c|c|c|c|}
\hline \multirow{2}{*}{$\begin{array}{c}\text { Dados Pessoais } \\
\text { Faixa Etária }\end{array}$} & \multicolumn{2}{|c|}{ Sexo masculino } & \multicolumn{2}{|c|}{ Sexo feminino } & \multirow[t]{2}{*}{$p$} \\
\hline & $\mathbf{n}$ & $\%$ & $\mathbf{n}$ & $\%$ & \\
\hline 60 a 69 anos & 42 & 51,9 & 76 & 55 & \\
\hline 70 a 79 anos & 29 & 35,8 & 35 & 25,4 & 0,167 \\
\hline A partir de 80 anos & 10 & 12,3 & 27 & 19,6 & \\
\hline Total & 81 & 100 & 138 & 100 & \\
\hline \multicolumn{6}{|l|}{ Zona de Residência } \\
\hline Zona Urbana & 69 & 89,6 & 121 & 90,9 & \\
\hline Zona rural & 8 & 10,4 & 11 & 9,1 & 0,618 \\
\hline Total & 77 & 100 & 132 & 100 & \\
\hline \multicolumn{6}{|l|}{ Etnia } \\
\hline Branca & 14 & 30,4 & 31 & 32,3 & \\
\hline Não branca & 32 & 69,6 & 65 & 67,7 & 0,824 \\
\hline Total & 46 & 100 & 96 & 100 & \\
\hline \multicolumn{6}{|l|}{ Escolaridade } \\
\hline Sem/Baixa Escolaridade* & 10 & 43,5 & 21 & 65,6 & \\
\hline Media/Alta escolaridade** & 13 & 56,5 & 11 & 34,4 & 0,102 \\
\hline Total & 23 & 100 & 32 & 100 & \\
\hline
\end{tabular}

Analfabetos, Ensino fundamental Completo e Incompleto*; Ensino médio completo e incompleto, Ensino superior completo e incompleto *; $\mathrm{p}$ - Teste Qui-Quadrado de Independência

Fonte: Adaptado do SINAN (2021).

Na Tabela 1 é possível observar-se que o sexo mais acometido é o feminino com 63,0\% e a faixa etária de 60 a 69 anos é a mais predominante, apresentado um percentual de 53,9\%. Ao aplicar o teste Qui-Quadrado entre as variáveis não foi possível verificar associação estatística entre as variáveis $(\mathrm{p}=0,167)$.

Dessa forma, nos estudos realizados por Oliveira et al (2018), na cidade de São Paulo, durante os anos de 2004 a 2006, dos 3.366 a maioria dos idosos também pertenciam ao sexo feminino, corroborando com nossos dados (Oliveira, Antunes \& Nicolina, 2018). Já no estado do Maranhão, em 2011 a 2015, entre os 21 idosos acometidos a idade que mais prevaleceu foi a partir de 80 anos com 42,9\% (n=9), contrariando os dados obtidos, no entanto outra pesquis a realizada no Brasil, entre 2007 e 2011, dos 3.728 idosos cerca de 53,1\% pertenciam a 60-69 anos, colaborando o estudo da Paraíba (Chaves et al., 2017).

$\mathrm{Na}$ literatura, é visto que as mulheres são as que mais consomem e as responsáveis no âmbito domiciliar pelo armazenamento dos medicamentos e isto contribui para uma maior automedicação, duas vezes mais do que os homens, pois apresentam mais sintomas como dores de cabeça, dores musculares e condições dolorosas crônicas, como enxaqueca e começam a fazer o uso de medicação muito cedo para o alívio de dores que aparecem na menstruação ou dismenorreia. Além do mais, apresentam mais tentativas de suicídio em comparação com os homens (Rangel \& Francelino, 2018; Arrais et al., 2016; Vieira \& Caveião, 2016).

Segundo Oliveira et al (2017) é de grande importância estudos que indiquem divergências nos perfis da saúde do homem e mulher, pois é possível identificar através dos mesmos variações das práticas relativas aos gêneros na compreensão de saúde-doença (Oliveira et al., 2017). Os idosos são o grupo etário que mais consomem medicamentos, como analgésicos gastrointestinais, antiinflamatórios, pomadas tópicas, ansiolíticos, hipertensivos, diabetes e entre outros (Gonçalves et al., 2017).

Com relação à zona de residência dos idosos, fica nítido que a maioria das notificações são daqueles residentes em 
zona urbana 89,6\% seguido dos pertencentes da zona rural 10,4\%, além disso, é importante destacar a quantidade de ignorados que apresentou um total de 6,9\%, dessa forma verifica-se que, as mulheres da cidade são as mais acometidas com 90,9\%, porém, não houve associação estatística entre as variáveis $(\mathrm{p}=0,618)$. Esse caráter urbano das intoxicações pode ser explicado pelo fato de que a maioria dos habitantes do estado da Paraíba serem residentes da zona urbana, o que permite uma maior facilidade de acesso dessas pessoas até as drogarias pertencentes a essa localidade (Brasil, 2020)

Diante do exposto, verifica-se que às localidades na zona rural são mais vulneráveis e proporcionam uma série de custos ao indivíduo, sejam aqueles relacionados à infraestrutura, como maior distância e dificuldade para acesso aos serviços públicos, entretanto, é importante ressaltar que, a maior dificuldade para acessar os serviços de saúde nas áreas rurais relaciona-se a uma menor disponibilidade de serviços de acompanhamento individual, além das dificuldades que os residentes dessa área encontram em se deslocar para outras cidades em busca de um melhor acompanhamento de saúde (Arruda, Maia \& Alves, 2018).

Ainda de acordo com a tabela 1, em relação à etnia dos idosos acometidos por intoxicação medicamentosa, evidenciase que aqueles não brancos são os que têm maior percentual com 68,3\% e os menos acometidos são da cor branca com 31,7\%, além disso, verifica-se 35,2\% da quantidade de casos foram ignorados. Ao aplicar o teste Qui-quadrado foi possível observar que não houve associação estatística entre as variáveis $(\mathrm{p}=0,824)$.

A maioria das notificações dos indivíduos serem não brancos pode ser atribuída ao fato de que, segundo o censo do IBGE, a maioria (57\%) dos idosos da Paraíba pertence a outras etnias como parda, preta e indígena (Brasil, 2020). Outra teoria pode advir de fatos históricos como o fim da escravidão, que deixou as populações pretas, pardas e indígenas com uma carência de políticas sociais, econômicas e com as piores condições de saúde, que refletem ainda nos tempos modernos (Oliveira et al., 2016). Destarte, a miscigenação e o critério auto referido adotado para classificar a cor são suscetíveis a influências culturais e subjetividade, o que dificulta a discussão de prováveis fatores sociodemográficos relacionado à quantidade de intoxicação (Tannous, 2018).

Com relação à escolaridade (Tabela 1) pode-se perceber que os idosos com sem/ baixa escolaridade são os mais acometidos com uma margem de $56,4 \%$, já os de média e alta escolaridade tiveram $43,6 \%$, em relação a quantidade de ignorados foi de $74,9 \%$, entretanto observa-se que não houve variação estatística entre as variáveis $(\mathrm{p}=0,102)$.

Este fato pode ser evidenciado pelo fato de que o um baixo grau de escolaridade pode refletir em um maior índice de casos, pois a desinformação e o desconhecimento sobre o uso correto de medicamentos pode acarretar no risco de intoxicações (Oliveira \& Suchara, 2014).

O SINAN apresenta na sua data base os tipos de circunstâncias que levaram a intoxicação medicamentosa nos idosos, que pode ser visto na Figura 3. 
Figura 3. Dados das Circunstâncias dos idosos acometidos por intoxicação medicamentosa na Paraíba, entre os anos de 2007 a 2017.

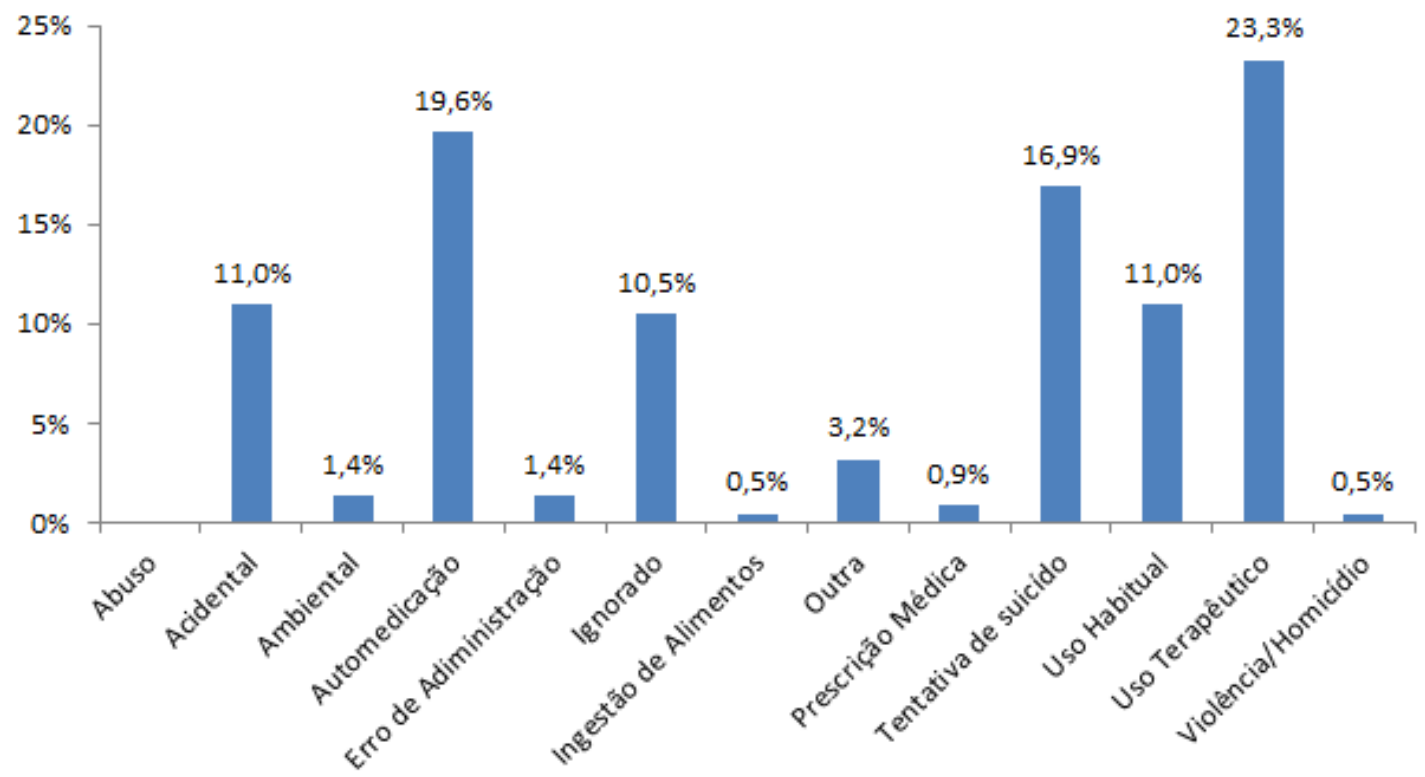

Fonte: Adaptado do SINAN (2021).

Segundo a Figura 3, as circunstâncias que mais levaram os idosos a intoxicação por medicamentos foram de três causas principais como uso terapêutico 23,3\%, automedicação 19,6\% e tentativas de suicídio 16,9\%.

Segundo Chaves et al (2017), as mulheres são as que mais se automedicam, por causa de fatores diários domiciliares e de trabalho, além de adotarem a prática de "farmácia caseira", que é composta de medicamentos, de venda livre e os de prescrição médica, levam ao abuso (Chaves et al., 2017). A associação entre o sexo e fatores que levaram a intoxicação medicamentosa nos idosos pode ser vista Tabela 2.

Tabela 2. Associação entre sexo e as circunstâncias dos acometidos por intoxicação medicamentosa na Paraíba, entre os anos de 2007 a 2017.

\begin{tabular}{ccccc|c}
\hline Circunstância & Masculino & $\mathbf{\%}$ & Feminino & $\boldsymbol{\%}$ & $\boldsymbol{p}$ \\
\hline Automedicação & 19 & 23,5 & 24 & 17,4 & \\
Uso terapêutico & 21 & 38,3 & 30 & 41,3 & \\
Tentativa de & 10 & 12,3 & 27 & 19,6 & 0,387 \\
$\quad$ suicídio & & & & & \\
Outra & 31 & 25,9 & 57 & 21,7 & \\
\hline Total & $\mathbf{8 1}$ & $\mathbf{1 0 0}$ & $\mathbf{1 3 8}$ & $\mathbf{1 0 0}$ & \\
\hline
\end{tabular}

P - Teste Qui-Quadrado de Indepedência. Fonte: Adaptado do SINAN (2021).

O sexo feminino foi o que mais sofreu intoxicação por uso terapêutico 41,3\% e tentativa de suicídio $19,6 \%$, na utilização de medicamentos, em relação aos homens. Porém, não houve diferença significativa entre as variáveis (p=0,387).

Segundo Vieira, Santa e Suchara (2015) a busca por métodos indolores, como uso de medicamentos, nas tentativas de suicídio é característico do público feminino, visto que são quatro vezes mais propensas a tentar suicídio do que os homens, porém as mulheres reconhecem mais cedo os sintomas da depressão, suicídio e doença mental e logo buscam ajuda seja através da religião ou com um profissional da saúde (Vieira, Santana, Suchara, 2015).

Vale salientar, que o Brasil é o quarto país em crescimento em suicídios da América Latina, por isso é de suma 
importância que o farmacêutico realize uma fiscalização rigorosa na dispensação de medicamentos de controles especial, visto que são os comumente envolvidos, preconizando a atenção farmacêutica através do acompanhamento farmacológico aos paciente que fazem uso desses medicamentos e promover a conscientização dos outros profissionais de saúde que atuam com pacientes que possuem perfis suicidas (Queiros et al., 2020).

A automedicação é o ato de consumir medicamentos sem orientação médica, que podem ocasionar problemas à saúde, com a intoxicação, sendo que 2014 cerca de 80 milhões de brasileiros eram adeptos (Macedo et al.,2016). Ela corresponde a 35 $\%$ da aquisição de medicamentos no Brasil e esse fato leva a 27\% das intoxicações e 16\% dos óbitos, contribuindo assim para 15 a $20 \%$ dos gastos hospitalares para lidar com estas complicações (Vieira \& Caveião, 2016). No entanto, instruções aos profissionais da área da saúde como farmacêuticos e balconistas de farmácia podem reduzir e tornar o uso de medicamentos mais racional e seguro (Macedo et al., 2016).

A evolução dos casos notificados por intoxicação medicamentosa em idosos pode ser verificada Tabela 3 .

Tabela 3. Evolução dos casos de idosos acometidos por Intoxicação medicamentosa na Paraíba, entre os anos de 2007 a 2017.

\begin{tabular}{ccc}
\hline Evolução & n & \% \\
\hline Cura sem Sequela & 159 & 72,6 \\
Cura com Sequela & 1 & 0,5 \\
Óbito & 2 & 0,9 \\
Perda de Seguimento & 1 & 0,5 \\
Ignorados & 56 & 25,5 \\
\hline Total & $\mathbf{2 1 9}$ & $\mathbf{1 0 0}$ \\
\hline
\end{tabular}

Fonte: Adaptado do SINAN (2021).

Analisando a Tabela 3, verifica-se que a maioria dos casos de intoxicação nos idosos obteve cura com 72,6 \% e 0,91\% dos indivíduos foram a óbito. No entanto $25,5 \%$ apresentavam-se ignorados.

A alta taxa de cura nos casos pode ter ocorrido por causa da assistência médica em tempo hábil, contudo o tratamento dos casos é feito através de tratamento de suporte, prevenção de absorção das substâncias tóxicas, fortalecimento de sua eliminação, uso de carvão ativado, imediata lavagem gástrica e o uso de antídotos que neutralizam o efeito do medicamento, essas formas contribuem para uma boa evolução clínica do paciente (Oliveira \& Suchara, 2014; Rangel \& Francelino, 2018).

Os medicamentos trazem uma boa relação custo-benefício, porém sua utilização de forma descontrolada se torna um grande agravo à saúde e a economia mundial, ademais possuir estoque de medicamentos em casa elevam as chances de automedicação e as tentativas de suicídio (Martins \& Oliveira, 2019).

As intoxicações medicamentosas podem ser influenciadas por prescrições médicas ilegíveis, inadequadas e falta de diálogo, sobre o uso do medicamento e histórico do paciente, assim trazem à tona a necessidade de orientação e educação dos profissionais de saúde (Vieira, Caveião, 2016).

Segundo Pereira, Ribeiro e Brito (2020), a presença do profissional farmacêutico é de suma importância frente as intoxicações por medicamentos, pois a atenção farmacêutica prestada promove a proteção e recuperação da saúde, buscando fornecer informações referente ao uso racional dos medicamentos através da educação em saúde. Portanto, apesar da assistência farmacêutica ser bastante essencial para diminuição dos casos de intoxicação, ela também é responsável por auxiliar da melhor forma a adesão de um tratamento correto a fim de proporcionar uma melhor qualidade de vida a coletividade (Gretzler et al., 2018).

Pode-se destacar que os usos de dados secundários são de suma importância, perante uma avaliação e interpretação adequada, para analisar as problemáticas enfrentadas pela saúde e criar e proporcionar estratégias viáveis nessa área (Prado et al., 2011). Porém, o estudo apresenta limitações devido à subnotificação e as informações consideradas ignoradas, 
necessitando de melhorias na coleta e preenchimento de dados.

\section{Conclusão}

No estado da Paraíba entre os anos de 2007 a 2017 foram notificados 219 casos de intoxicação exógena por medicamentos em idosos, apresentando o ano de 2014 como mais prevalente e a cidade que apresentou mais notificações foi a capital João Pessoa.

O perfil epidemiológico de acometidos por intoxicação medicamentosa foi majoritariamente de indivíduos do sexo feminino, com 60 a 64 anos de idade, de cor parda, residentes de zona urbana, com ensino fundamental incompleto, sendo as circunstâncias que mais levaram a intoxicação foram o uso terapêutico, automedicação e a tentativa de suicídio, contudo, a maioria dos casos relatados foram curados.

Assim, a obtenção desses dados pode ajudar na criação de políticas públicas ou indicadores, em nível municipal e estadual, com o intuito de atender a população mais acometida, e fomentar a criação de pesquisas semelhantes em outros estados e municípios. Vale ressaltar a importância do profissional farmacêutico na promoção à saúde, visto que o exercício clínico farmacêutico, desempenhado através da prática do cuidado farmacêutico pode contribuir para a diminuição de intoxicação medicamentosa na população e especialmente em idosos, que são polimedicados.

Por fim, como os idosos e as crianças são os grupos mais afetados pelas intoxicações com medicamentos, sugerimos que sejam feitos trabalhos epidemiológicos com o público infantil no estado da Paraíba.

\section{Referências}

Arrais, P. S. D., Fernandes, M. E. P., Pizzol, T. S. D., Ramos, L. R., Mengue, S. S., Luiza, V. L., Tavares, N. U. L., Farias, M. R., Oliveira, M. A., \& Bertoldi, A. D. (2016). Prevalência da automedicação no Brasil e fatores associados. Revista de Saúde Pública, $50(2), 13$.

Arruda, N. M., Maia, A. G., \& Alves, L. C. (2018). Desigualdade no acesso á saúde entre as áreas urbanas e rurais do Brasil: uma decomposição de fatores entre 1998 a 2008. Caderno de Saúde Pública, 34(6), 2-14.

Barros Lima, A. M. E., Ferreira, R. C., dos Santos-Neto, P. E., Rodrigues, C. A. Q., de Pinho Veloso, D. N., Maia, J., \& Costa, D. C. (2013). Delineamentos de estudos epidemiológicos e não epidemiológicos da área da saúde: uma revisão de literatura. Revista Unimontes Científica, 15(2), 64-80.

Brasil, Departamento de Informática do SUS (DATASUS) (2020). Informações Epidemiológicas e Morbilidade: banco de dados. https://datasus.saude.gov.br/informacoes-de-saude-tabnet/

Brasil, Instituto Brasileiro de Geografia Estatística (IBGE) (2020). Paraíba. https://cidades.ibge.gov.br/brasil/pb/panorama

Bitter, K., Schlender, J. F., \& Woltersdorf, R. (2016). Drug-drug interactions in the elderly: Which ones really matter? Der Internist, 57(7):728-734.

Conselho Federal de Farmácia (CFF) (2019). Dados 2018. https://www.cff.org.br/pagina.php?id=801\&menu=801\&titulo=Dados+2018

Chaves, L. H. S., Viana, A. C., Júnior, W. P. M., Lima, A., \& Carvalho, L. (2017). Intoxicação exógena por medicamentos: aspectos epidemiológicos dos casos notificados entre 2011 e 2015 no Maranhão. Revista Ciência \& Saberes-Facema, 3(2), 477-482.

Gonçalves, C. A., Gonçalves, C. A., Santos, V. A., Sarturi, L., \& Júnior, A. T. T. (2017). Intoxicação medicamentosa. Revista Científica da Faculdade de Educação e Meio Ambiente, 8(1), 135-143.

Gotadelo, D. R., Fonseca, L. S., Masson, E. R., Lopes, L. N., Toledo V. C., Faioli, M. A., Meira, A. L. M., Costa, C. K. M., \& Andrade, R. B. L. (2014). Prevalência e fatores associados a potenciais interações medicamentosas entre idosos em um estudo de base populacional. Revista Brasileira de Medicina de Família e Comunidade, 9(31), 111-118.

Gretzler, V. S., Rodrigues, A. S., Vargas, D. A., Pereira, H. C., \& Júnior, A. T. T. (2018). Atuação do farmacêutica na URM e na prevenção de intoxicação medicamentosa. Revista Científica FAEMA, 9, 547-550.

Instituto de Desenvolvimento Municipal e Estadual. Anuário estatístico do estado da Paraíba 2014 (2014) http://ideme.pb.gov.br/servicos/anuarios-online

Lima, H. S. M., Teixeira, A. P. C., \& Oliveira, F. S. (2018). Uso de benzodiazepínicos em idosos: uma revisão integrativa. Revista Educação Ciência e Saúde, $5(1), 70-88$.

Maia, B. C., Viana, P. S., Arantes P. M. M., \& Alencar M. A. (2011) Consequências das quedas em idosos vivendo na comunidade. Revista brasileira de geriatria e gerontologia, 14(2), 381-393. 
Macedo. G. R., Carmo, B. B., Castro, G. F. P., \& Correa, J. B. (2016). O poder do marketing no consumo excessivo de medicamentos no Brasil. Revista Transformar, 9:114-128.

Martins, A. O. \& Oliveira, D. H. Perfil de intoxicação e óbito por medicamento no brasil: uma revisão sistemática. International Journal of Development Research, 9(11), 31883-31887.

Mastroianni, P. D. C., Lucchetta, R. C., Sarra, J. D. R., \& Galduróz, J. C. F. (2011). Estoque doméstico e uso de medicamentos em uma população cadastrada na estratégia saúde da família no Brasil. Revista Panamericana de Salud Publica, 29, 358-364.

Medeiros, K. K. M., Soares, P. M., Saatkamp, J. D. A., Tavares, G. M. S., \& Farias, F. M. (2012). Uso de medicamentos como um fator de risco para fraturas por quedas em idoso. Anais do Salão Internacional de Ensino, Pesquisa e Extensão, 4 (2).

Mendes, J. L. V., Silva, S. C., Silva, G. R., \& Santos, N. A. R. (2018). O aumento da População Idosa no Brasil e o envelhecimento nas últimas década: Uma revisão da literatura. Revista Educação, Meio Ambiente e Saúde (REMAS), 8(1), 13-26.

Miranda, G. M. D., Mendes, A. C. G. \& Silva A. L. A. (2019). O envelhecimento populacional brasileiro: desafios e consequências sociais atuais e futuras. Revista Brasileira de Geriatria e Gerontologia, 19(3), 507-519.

Mota, D. M., Melo J. R. R., Freitas D. R. C. D., \& Machado, M. (2012). Perfil da mortalidade por intoxicação com medicamentos no Brasil, 1996-2005: retrato de uma década. Ciência \& Saúde Coletiva, 17, 61-70.

Oliveira, B. L. C. A., Silva, A. M. D., Rodrigues, L. D. S., \& Rêgo, A. S. (2016). O uso da modelagem com equações estruturais na análise da influência da cor/raça e status socioeconômicos na saúde de idosos Brasileiros. Revista Brasileira de Ciências da Saúde, 20(2), $149-156$.

Oliveira, D. H. \& Suchara, E. A. (2014). Intoxicações medicamentosas em hospital público de Barra do Garças-MT. No período de 2006 a 2009. Revista de Ciências Médicas e Biológicas, 13(1), 55-59.

Oliveira, J., Antunes, J. L. F., \& Nicolina, L. (2018). Caracterização das internações medicamentosas, São Paulo, 2004 a 2006. Archives of Health Investigation, 7(6), 237-242.

Oliveira, J. F. M., Wagner, G. A., Lieber, N. S. R., \& Antunes, J. L. (2017). Tendência da mortalidade por intoxicação medicamentosa entre gêneros e faixa etárias no Estado de São Paulo, Brasil, 1996-2012. Ciência \& Saúde Coletivo, 22(10), 3381-3391.

Organização Mundial da Saúde (OMS) (2015). Resumo: relatório mundial de envelhecimento e saúde.

Prado, T. N., Caus, A. L., Marques, M., Maciel, E. L., Golub, J. E., \& Miranda, A. E. (2011). Perfil epidemiológico de pacientes adultos com tuberculose e AIDS no estado do Espírito Santo: relacionamento dos bancos de dados de tuberculose e AIDS. Jornal Brasileiro de Pneumologia, 37(1), 93-99.

Pereira, C. E. D., Ribeiro, R. L. C. \& Brito, M. H. R. M. (2020). Perfil das principais intoxicações exógenas no estado do Piauí: análise epidemiológica de uma década. Research, Society and Development, 9(3), 30.

Pereira, M. D., Conceição, S. B., Mariúba, G. B., Santos, N. S., \& Rebelo, M. A. (2019). Envelhecimento populacional com foco no uso racional de medicamentos: O papel do farmacêutica. Revista InterSaúde, 1(1), 37-46.

Perissé, C. \& Marli, M. (2019). Idosos devem identificar caminhos para uma melhor idade. Revista Retratos. https://censo2020.ibge.gov.br/2012-agencia-denoticias/noticias/24036-idosos-indicam-caminhos-para-uma-melhor-idade.html

Queiros, A. J. C., Leite, R. S., Yoshida, E. H., Estanagel, T. H. P., Pereira, M. D., \& Santos, N. S. (2020). Perfil de suicídio por sobredose intencional de medicamentos. Revista InterSaúde, 1(2), 79-88.

Ramos, L. R., Tavares, N. U. L, Bertoldi, A. D., Farias, M. R. L., Oliveira, M. A., Luiza, V. L., Pizzol, T. S. D., Arrais, P. S. D, \& Mengue, S. S. (2016). Polymorbidity in Older Adults in Brazil: a public health challenge. Revista de Saúde Pública, 50(2), 9.

Rangel, N. \& Francelino, E. V. (2018). Caracterização do Perfil das Intoxicações Medicamentosas no Brasil, durante 2013 a 2016. Id online Revista de Psicologia, 42, 121-135.

Santos, I. S. \& Santos, R. C. (2019). Prevenção de Fraturas em Idosos. Monografia (Graduação em Enfermagem) - Centro Universitário do Planalto Central Apparecido dos Santos-Uniceplac.

Silva, H. O. N., Silva, E. L., Mariz, S. R., Bragagnolli, G., \& Fook, S. M. L. (2015). Perfil das intoxicações notificados na Paraíba de 2009 a 2013. Revista Cereus, 7(2), 78-87.

Silva, R., Schmidt, O. F., \& Silva, S. (2012). Polifarmácia em geriatria. Revista de AMRIGS, 56(2), 164-174.

Tannous, I. P. (2018). Perfil Epidemiológico e Geográfico da Infecção pelo Vírus da dengue em um município do Sudoeste de Goiás: Um estudo transversal. Dissertação (Mestrado em Ciências Aplicadas à Saúde) - Universidade Federal de Goiás, JATAÍ.

Vieira, D. M. \& Caveião, C. (2016). Perfil das intoxicações medicamentosas no estado de São Paulo no Período de 1999 a 2012 na perspectiva da vigilância sanitária. Revista Saúde e Desenvolvimento, 9(5), 119-141.

Vieira, L. P., Santana, V. T. P. \& Suchara E. A. (2015). Caracterização de tentativas de suicídios por substâncias exógenas. Cadernos de Saúde Coletiva, $23(2), 118-123$ 